\title{
Asymptotic and Non-asymptotic Confidence Regions in Closed Loop Model Validation
}

\author{
Hong Wang-Jian ${ }^{1}$, Tang De-zhi ${ }^{2}$ \\ ${ }^{1}$ School of Electronic Engineering and Automation, Jiangxi University of Science and Technology, Ganzhou, China \\ ${ }^{2}$ School of Electrical and Information Engineering, Anhui University of Technology, Ma-an-shan, China
}

Email address:

wangjianhong198019@aliyun.com (Hong Wang-jian)

\section{To cite this article:}

Hong Wang-Jian, Tang De-zhi. Asymptotic and Non-asymptotic Confidence Regions in Closed Loop Model Validation. Advances in Wireless Communications and Networks. Vol. 3, No. 6, 2017, pp. 75-83. doi: 10.11648/j.awcn.20170306.11

Received: October 5, 2017; Accepted: November 17, 2017; Published: December 5, 2017

\begin{abstract}
In this paper one way is proposed to construct asymptotic and non-asymptotic confidence regions in the problem of closed loop model validation deeply. These two asymptotic and non-asymptotic confidence regions correspond to the infinite and finite data points. Firstly one asymptotic confidence region is derived from some statistical properties on noise. The uncertainties bound of the model parameter is constructed in the probability sense by using the inner product form of the asymptotic covariance matrix, then a new technique for estimating bias and variance contributions to the model error is suggested. Secondly we modify sign perturbed sums (SPS) method to construct non-asymptotic confidence regions under a finite number of data points, where some modifications are studied for closed loop system. Finally the simulation example results confirm the identification theoretical results.
\end{abstract}

Keywords: Closed Loop Identification, Model Structure Validation, Asymptotic Region, Non-asymptotic Region

\section{Introduction}

The automatic control system includes two basic structures: one open loop and other closed loop. As there does not exist any feedback in open loop structure, so the plant output affects the input less. And in closed open structure, the error signals coming from the input and feedback output generate one correction action and make the output converge to some given value. The essence of closed loop system is to decrease the error by using the negative feedback function, and correct the deviation from the given value automatically. As the closed loop structure can suppress the errors coming from the internal or external disturbances, so for many industrial production processes, safety and production restrictions are strong reasons for not allowing control experiments in open-loop and the closed loop is most needed in all of our engineering.

Generally two strategies are used to design the controller in closed loop, i.e. model based design and direct data driven design. The primary step of model based design is to construct the plant model and apply this mathematical model in the process of designing controller. Conversely in the direct data driven method, the modeling process is not needed and the controller is directly designed by using the input-output data. Now as the first model based design strategy is more applied, so we do much research on system identification to identify the plant model. The whole theory of system identification can be divided into four categories, i.e. experiment design [1], model structure selection [2], model parameter identification [3] and model structure validation test [4]. Further the more research is concerned on the first three categories. To be the author's knowledge, the study on model structure validation under closed loop condition is very little.

There are three common identification methods for closed loop system identification, i.e. direct approach, indirect approach and joint input-output approach, where the feedback is neglected in direct approach and the plant model is identified directly using the input-output data. In the indirect approach for closed loop system identification, the feedback effect is considered and the input-output from the whole closed loop condition are used to identify the plant model. The joint input-output approach is very similar to indirect approach. In [5], three methods are presented to identify closed loop system. In [6], researches on the system identification theory are introduced in time domain. Similarly the frequency domain system identification is given in [7]. A 
new virtual closed loop method for closed loop identification is proposed in [8]. In [9], one projection algorithm is proposed on the basis of the prediction error recursive method. In [10], when many inputs exist in closed loop, whether can closed loop be identified with parts of the inputs controlled? The relationship between closed loop identification and closed loop control are obtained in [11]. In [12], the linear matrix inequality is used to describe the problem of optimal input design in closed loop. Further the least cost identification experiment problem is analyzed in [13]. The power spectral of the input signal is considered to be an objective function and the accuracy of the parameter estimations is the constraints [14]. In [15], H-infinity norm from robust control is introduced to be the objective function in the optimal input design problem. Based on the $\mathrm{H}$-infinity norm, the uncertainty between the identified model and nominal model is measured and the optimal input is chosen by minimizing this uncertainty [16]. The selection of the optimal input can also be determined from the point of asymptotic behavior about the parameter estimation [17]. The Persistent excitation input in closed loop is analyzed and we obtain some conditions about how to obtain persistent excitation [18]. In [19] the problem of how to apply closed loop identification into adaptive control is solved, so that bias and covariance terms are isolated separately. All above results hold when the number of the observed signals will convergent to infinity.

There are little papers about model structure validation test now. Only in [6] and [19], model structure validation in open loop identification has been presented and the standard cross correlation test is proposed to test the confidence region of the cross variance matrix between the prediction error and input from the probabilistic sense. Because of the simple structure of open loop, the process of deriving the covariance matrix is very easy. For model structure validation in engineering, the more effective strategy is to do one similar experiment again. After exciting the formal system with a group of new inputs, we compare if the actual output is consistent with the identified output. Although this test is simple, we can not analyze the accuracy and credibility of the identified model. In our paper [20], two probabilistic model uncertainties and optimum input filter are derived from some statistical properties of the parameter estimation. The probabilistic bounds and optimum input filter are based on an asymptotic normal distribution of the parameter estimator and its covariance matrix, which was estimated from sampled data.

Using some results from our former paper [20], in this paper we continue to study the problem of model structure validation for closed loop system identification. So in order to reflect the identification accuracy, here we apply the statistical probability framework to derive the variance matrix of the unknown parameters firstly. This variance matrix is decomposed into one inter product form which is used to construct one uncertainty bound about the unknown parameter estimation. This uncertainty bound is called by confidence region and it constitutes the guaranteed confidence region with respect to the model parameter estimation under closed loop condition. As statistical probability framework needs infinite data points, but in practice, we only have a finite number of data points and limited statistical knowledge about the noise. So to relax this strict condition on the number of data points, we introduce sign-perturbed sums (SPS) to construct non-asymptotic confidence region. Due the original SPS method is suited for linear regression model, it can not be appropriate for our closed loop system. This paper introduces and analyzes the modified SPS method for closed loop system. This confidence region constructed by the modified SPS method contains the true parameters with a user chosen exact probability for a finite data points. To the best of our knowledge, the asymptotic confidence region or non-asymptotic confidence region correspond to only linear regression model, not our closed loop system. To achieve our ultimate goal of closed loop model validation, much work is achieved to extend the existing theories and modify them to be suitable for closed loop system.

\section{Problem Description}

Consider the following actual closed loop system with output feedback in Figure 1.

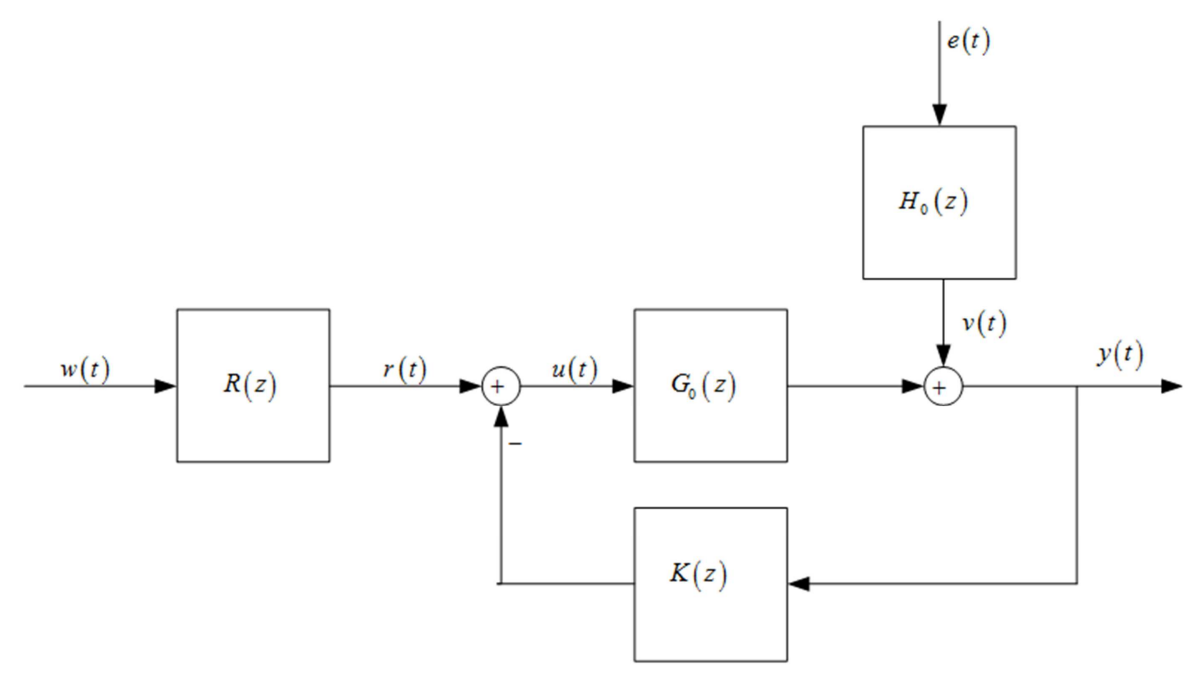

Figure 1. Structure of the closed loop system. 
where $G_{0}(z)$ is the true plant model; $H_{0}(z)$ is the noise filter, they are all linear time invariant transfer functions. $K(z)$ is a stable linear time invariant controller, here we assume this controller is priori known. The excited signal $r(t)$ and external disturbance $e(t)$ are assumed to be uncorrelated, $e(t)$ is a white noise with zero mean value and variance $\lambda_{0} . v(t)$ is a colored noise which can be obtained by passing white noise $e(t)$ through the noise filter $H_{0}(z)$. $u(t)$ and $y(t)$ are the input-output signals corresponding to plant model $G_{0}(z)$.

Rewriting excited signal $r(t)$ as white noise $w(t)$ passing through shaping filter $R(z) . R(z)$ is the power spectrum factor with stable non-minimal phase of excited signal $r(t) . z$ is the delay operator, it means that $z u(t)=u(t+1)$.

As $r(t)=R(z) w(t)$, the power spectrum density of excited signal is given as.

$$
\phi_{r}(\omega)=R(z) R^{*}(z) \phi_{w}(\omega)=|R(z)|^{2}
$$

In closed loop system structure, through some computations, we derive the transfer function form.

$$
y(t)=G_{0}(z) R(z) w(t)-G_{0}(z) K(z) y(t)+H_{0}(z) e(t)
$$

Continuing to do some computations and we get.

$$
\left\{\begin{array}{l}
y(t)=\frac{G_{0}(z) R(z)}{1+G_{0}(z) K(z)} w(t)+\frac{H_{0}(z)}{1+G_{0}(z) K(z)} e(t) \\
u(t)=\frac{R(z)}{1+G_{0}(z) K(z)} w(t)-\frac{K(z) H_{0}(q)}{1+G_{0}(z) K(z)} e(t)
\end{array}\right.
$$

To simplify the analysis process, define the sensitivity function as.

$$
S_{0}(z)=\frac{1}{1+G_{0}(z) K(z)}
$$

The output of closed loop system can be written as.

$$
\left\{\begin{array}{l}
y(t)=G_{0}(z) R(z) S_{0}(z) w(t)+H_{0}(z) S_{0}(z) e(t) \\
u(t)=R(z) S_{0}(z) w(t)-K(z) H_{0}(z) S_{0}(z) e(t)
\end{array}\right.
$$

As now our goal is not emphasized on identification method, but on the model structure validation, so the only simple direct approach is used to describe the basic ideas.

\section{Asymptotic Confidence Region}

Introduce the unknown parameter vector in closed loop system, the parameterized form given by.

$$
\left\{\begin{array}{l}
y(t, \theta)=\frac{G(z, \theta) R(q)}{1+G(z, \theta) K(z)} w(t)+\frac{H(z, \theta)}{1+G(z, \theta) K(z)} e(t) \\
u(t, \theta)=\frac{R(z)}{1+G(z, \theta) K(z)} w(t)-\frac{K(z) H(z, \theta)}{1+G(z, \theta) K(z)} e(t)
\end{array}\right.
$$

where $\theta$ denotes the unknown parameter vector, it exists in the parameterized plant model $G(z, \theta)$ and noise model $H(z, \theta)$ respectively. The goal of closed loop identification is to identify the unknown parameter vector $\hat{\theta}_{N}$ from one given input-output data set $Z^{N}=\{y(t), u(t)\}_{t=1}^{N}$, where $N$ denotes the number of total observed data.

According to equation (5), the prediction of $y(t, \theta)$ can be calculated as the one step ahead prediction.

$$
\begin{aligned}
& \hat{y}(t, \theta)=\left[\frac{H(z, \theta)}{1+G(z, \theta) K(q)}\right]^{-1} \frac{G(z, \theta) R(z)}{1+G(z, \theta) K(z)} w(t) \\
& +\left[1-\left[\frac{H(z, \theta)}{1+G(z, \theta) K(z)}\right]^{-1}\right] y(t) \\
& \quad=\frac{1+G(z, \theta) K(z)}{H(z, \theta)} \times \frac{G(z, \theta) R(z)}{1+G(q, \theta) K(z)} w(t) \\
& \quad+\left[1-\frac{1+G(z, \theta) K(z)}{H(z, \theta)}\right] y(t) \\
& \quad=\frac{G(z, \theta) R(z)}{H(z, \theta)} w(t)+\frac{H(z, \theta)-1-G(z, \theta) K(z)}{H(z, \theta)} y(t)
\end{aligned}
$$

Computing the one step ahead prediction error or residual, now it becomes.

$$
\begin{aligned}
& \varepsilon(t, \theta)=y(t)-\hat{y}(t, \theta) \\
& \quad=\frac{1+G(z, \theta) K(q)}{H(z, \theta)}\left[y(t)-\frac{G(z, \theta) R(z)}{1+G(z, \theta) K(z)} w(t)\right]
\end{aligned}
$$

In the standard prediction error algorithm, when using the input-output data $Z^{N}=\{y(t), u(t)\}_{t=1}^{N}$ with the data number $N$, the parameter vector is identified by.

$$
\begin{aligned}
& \hat{\theta}_{N}=\underset{\theta}{\arg \min } V_{N}\left(\theta, Z^{N}\right)=\underset{\theta}{\arg \min } \frac{1}{N} \sum_{t=1}^{N} \varepsilon^{2}(t, \theta) \\
& V_{N}\left(\theta, Z^{N}\right)=\frac{1}{N} \sum_{t=1}^{N} \varepsilon^{2}(t, \theta)
\end{aligned}
$$

Defining the asymptotic limit parameter estimate $\theta^{*}$ as.

$$
\theta^{*}=\underset{\theta}{\arg \min } \lim _{N \rightarrow \infty} E\left\{V_{N}\left(\theta, Z^{N}\right)\right\}
$$

where $E$ denotes the expectation operator. In the common identification process, assume that there always exists one true 
parameter vector $\theta_{0}$ such that.

$$
G\left(z, \theta_{0}\right)=G_{0}(z), H\left(z, \theta_{0}\right)=H_{0}(z)
$$

This assumption shows that the identified model is contained in the considered model set. Based on some results from [6], the asymptotic matrix of the parameter estimate is get.

$$
\operatorname{cov} \hat{\theta}_{N}=\lambda_{0}\langle\varphi, \varphi\rangle^{-1}
$$

where $\langle\varphi, \varphi\rangle$ denotes some inter product operator, $\varphi$ is the negative gradient of the predictor error, i.e. it can be computed from:

$$
\varphi(t, \theta)=-\frac{\partial \varepsilon(t, \theta)}{\partial \theta}=\frac{\partial \hat{y}(t, \theta)}{\partial \theta}
$$

Next we give the calculation process of the negative gradient of the predictor error under closed loop condition below. As equation (11) is a basic formula in studying asymptotic analysis, we substitute equation (5) into equation (6) to get.

$$
\begin{aligned}
& \hat{y}(t, \theta)=\frac{G(z, \theta) R(z)}{H(z, \theta)} w(t) \\
& +\frac{H(z, \theta)-1-G(z, \theta) K(z)}{H(z, \theta)} y(t) \\
& =\frac{G(z, \theta) R(q)}{H(z, \theta)} w(t)+\frac{H(z, \theta)-1-G(z, \theta) K(z)}{H(z, \theta)} \\
& \times \underbrace{\left[\frac{G(z, \theta) R(z)}{1+G(z, \theta) K(z)} w(t)+\frac{H(z, \theta)}{1+G(z, \theta) K(z)} e(t)\right]}_{y(t)} \\
& =\frac{G(z, \theta) R(z)}{H(q, \theta)} w(t) \\
& +\frac{H(z, \theta)-1-G(z, \theta) K(z)}{H(z, \theta)} \times \frac{G(z, \theta) R(z)}{1+G(z, \theta) K(z)} w(t) \\
& +\frac{H(z, \theta)-1-G(z, \theta) K(z)}{H(z, \theta)} \times \frac{H(z, \theta)}{1+G(z, \theta) K(z)} e(t) \\
& =\frac{G(z, \theta) R(z)}{H(z, \theta)} w(t) \\
& +\frac{H(z, \theta)-1-G(z, \theta) K(z)}{1+G(z, \theta) K(z)} \frac{G(z, \theta) R(z)}{H(z, \theta)} w(t) \\
& +\frac{H(z, \theta)-1-G(z, \theta) K(z)}{1+G(z, \theta) K(z)} e(t) \\
& =\frac{G(z, \theta) R(z)}{1+G(z, \theta) K(z)} w(t) \\
& +\frac{H(z, \theta)-1-G(z, \theta) K(z)}{1+G(z, \theta) K(z)} e(t)
\end{aligned}
$$

where in the third equity of equation (13), we use the follow computation process.

$$
\begin{aligned}
& \frac{1}{H(z, \theta)}+\frac{H(z, \theta)-1-G(z, \theta) K(z)}{H(z, \theta)(1+G(z, \theta) K(z))} \\
& =\frac{1+G(z, \theta) K(z)+H(z, \theta)-1-G(z, \theta) K(z)}{H(z, \theta)(1+G(z, \theta) K(z))} \\
& =\frac{H(z, \theta)}{H(z, \theta)(1+G(z, \theta) K(z))}=\frac{1}{1+G(z, \theta) K(z)}
\end{aligned}
$$

Substituting equation (13) into equation (7) and computing the partial derivative operations with respect to unknown parameter vector $\theta$, and then we have.

$$
\begin{aligned}
\frac{\partial \varepsilon(t, \theta)}{\partial \theta}=\frac{G^{\prime}(\theta) K H(\theta)-[1+G(\theta) K] H^{\prime}(\theta)}{H(\theta)} & \times \frac{1}{1+G(\theta) K} e(t)-\frac{1}{H(\theta)} \\
& \times \frac{G^{\prime}(\theta) R[1+G(\theta) K]-G(\theta) R G^{\prime}(\theta) K}{1+G(\theta) K} w(t) \\
= & {\left[\frac{G^{\prime}(\theta) K}{H(\theta)[1+G(\theta) K]}-\frac{H^{\prime}(\theta)}{H(\theta)}\right] e(t) } \\
& -\left[\frac{G^{\prime}(\theta) R}{H(\theta)}-\frac{G(\theta) R G^{\prime}(\theta) K}{H(\theta)[1+G(\theta) K]}\right] w(t)
\end{aligned}
$$

where $G^{\prime}(\theta)$ denotes the partial derivative operation with respect to $\theta$, and the delay operator $q$ are all ignored to simply the derivations.

Using the uncorrelated assumption between white noise $e(t)$ and $w(t)$, i.e. it holds that.

$$
E e(t) w^{T}(t)=0
$$

putting ahead one $H(\theta)$ in equation (9), and rewriting it as.

$$
\begin{aligned}
\frac{\partial \varepsilon(t, \theta)}{\partial \theta} & =\frac{1}{H(\theta)}\left(\left[G^{\prime}(\theta) K S(\theta)-H^{\prime}(\theta)\right] e(t)\right. \\
& \left.-\left[G^{\prime}(\theta) R-G(\theta) R G^{\prime}(\theta) S(\theta) K\right] w(t)\right)
\end{aligned}
$$

where we use the parameterized sensitivity function.

$$
S(\theta)=\frac{1}{1+G(\theta) K}
$$

As the following equality holds.

$$
R-G(\theta) R K S(\theta)=\frac{R}{1+G(\theta) K}=R S(\theta)
$$

Rewriting equation (15) as the following matrix form. 


$$
\begin{aligned}
& \frac{\partial \varepsilon(t, \theta)}{\partial \theta}=\frac{1}{H(\theta)} \\
& \times\left[\begin{array}{ll}
G^{\prime}(\theta) & H^{\prime}(\theta)
\end{array}\right]\left[\begin{array}{cc}
K S(\theta) H(\theta) & R S(\theta) \\
-1 & 0
\end{array}\right]\left[\begin{array}{l}
e(t) \\
w(t)
\end{array}\right]
\end{aligned}
$$

According to equation (11), the asymptotic covariance matrix is that..

$$
\begin{aligned}
& P_{\theta}=\operatorname{cov} \hat{\theta}_{N}=\lambda_{0}\left[E \varphi\left(t, \theta_{0}\right) \varphi^{T}\left(t, \theta_{0}\right)\right]^{-1}=\langle\phi, \phi\rangle^{-1} \\
& \phi=\frac{1}{\sqrt{\lambda_{0}} H_{0}}\left[\begin{array}{ll}
G^{\prime}\left(\theta_{0}\right) & H^{\prime}\left(\theta_{0}\right)
\end{array}\right]\left[\begin{array}{cc}
\sqrt{\lambda_{0}} K S_{0} H_{0} & R S_{0} \\
-\sqrt{\lambda_{0}} & 0
\end{array}\right]
\end{aligned}
$$

where $G^{\prime}(\theta)$ and $H^{\prime}(\theta)$ are given as respectively.

$$
G^{\prime}(\theta)=\frac{\partial G(\theta)}{\partial \theta}, \quad H^{\prime}(\theta)=\frac{\partial H(\theta)}{\partial \theta}
$$

On basis of equation (21), we have the asymptotic result.

$$
\hat{\theta}_{N} \stackrel{N \rightarrow \infty}{\rightarrow} \theta_{0}
$$

It shows that the parameter estimator $\hat{\theta}_{N}$ will converge to its limit $\theta_{0}$, and further $\hat{\theta}_{N}$ will asymptotically converge $(N \rightarrow \infty)$ to normally distributed random variable with mean $\theta_{0}$ and variance $P_{\theta}$.

$$
\sqrt{N}\left(\hat{\theta}_{N}-\theta_{0}\right) \rightarrow \mathbb{N}\left(0, P_{\theta}\right) \text {, as } N \rightarrow \infty
$$

This asymptotic result can be rewritten in a quadratic form, and then we get one $\lambda^{2}$ distribution.

$$
N\left(\hat{\theta}_{N}-\theta_{0}\right)^{T} P_{\theta}^{-1}\left(\hat{\theta}_{N}-\theta_{0}\right) \stackrel{N \rightarrow \infty}{\rightarrow} \lambda_{n}^{2}
$$

Where $n$ is the number of degrees of freedom in the $\lambda^{2}$ distribution, being equal to the dimension of the parameter vector. Equation (25) implies that the random variable $\hat{\theta}_{N}$ satisfies one uncertainty bound.

$$
\begin{aligned}
& \hat{\theta}_{N} \in D\left(\alpha, \theta_{0}\right) \\
& D\left(\alpha, \theta_{0}\right)=\left\{\theta_{0} / N\left(\theta-\theta_{0}\right)^{T} P_{\theta}^{-1}\left(\theta-\theta_{0}\right) \leq \lambda_{n, \alpha}^{2}\right\}
\end{aligned}
$$

with $\lambda_{n, \alpha}^{2}$ corresponding to a probability level $\alpha$ in $\lambda_{n}^{2}$ distribution, but now in order to quantity the uncertainty on $\theta_{0}$ rather than $\hat{\theta}_{N}$, it holds that for every realization of $\hat{\theta}_{N}$.

$$
\hat{\theta}_{N} \in D\left(\alpha, \theta_{0}\right) \Leftrightarrow \theta_{0} \in D\left(\alpha, \hat{\theta}_{N}\right)
$$

It signifies that.

$$
\begin{aligned}
& \theta_{0} \in D\left(\alpha, \hat{\theta}_{N}\right) \text { with probability } \alpha \\
& D\left(\alpha, \hat{\theta}_{N}\right)=\left\{\theta / N\left(\hat{\theta}_{N}-\theta\right)^{T} P_{\theta}^{-1}\left(\hat{\theta}_{N}-\theta\right) \leq \lambda_{n, \alpha}^{2}\right\}
\end{aligned}
$$

Equations (26) and equation (28) give the confidence regions of unknown parameter estimator under closed loop condition. The probability level of the event $\hat{\theta}_{N} \in D\left(\alpha, \theta_{0}\right)$ holds is at least $\alpha$.

\section{Non-asymptotic Confidence Region}

Both confidence regions (26) and equation (28) are guaranteed only asymptotically when the number of measured data points tends to infinity, i.e. $N \rightarrow \infty$. But in practice, a finite number of measured data points and limited statistical knowledge about the noise are given, this fact strongly restricts above asymptotic confidence region. In this section, we use Sign-Perturbed Sums (SPS) method to construct non-asymptotic confidence region under relaxed statistical assumption on noise. SPS method is proposed for linear regression model in [21], and its advantage is that confidence region obtained by SPS method has exact confidence probability, i.e. it contains the true parameter with a user-chosen exact probability as the number of measured data points is finite. Now SPS process is introduced into model structure validation for closed loop system, i.e. we extend the linear regression model in [21], [22] to closed loop system and explain how SPS can be applied to construct guaranteed finite sample confidence region for closed loop system.

For the sake of completeness, from equation (6), we rewrite it as that.

$$
\begin{aligned}
\hat{\theta}_{N} & =\underset{\theta}{\arg \min } V_{N}\left(\theta, Z^{N}\right)=\underset{\theta}{\arg \min } \frac{1}{N} \sum_{t=1}^{N} \varepsilon^{2}(t, \theta) \\
& =\underset{\theta}{\arg \min } \frac{1}{N} \sum_{t=1}^{N}[y(t)-\hat{y}(t, \theta)]^{2}
\end{aligned}
$$

and the negative gradient of the predictor error

$$
\varphi(t, \theta)=-\frac{\partial \varepsilon(t, \theta)}{\partial \theta}=\frac{\partial \hat{y}(t, \theta)}{\partial \theta}
$$

Using the necessary condition on equation (8) to obtain one normal equation.

$$
\begin{aligned}
& \frac{1}{N} \sum_{t=1}^{N} \varphi(t, \theta) \mathcal{\varepsilon}(t, \theta)= \\
& \frac{1}{N} \sum_{t=1}^{N} \varphi(t, \theta)[y(t)-\hat{y}(t, \theta)]=0
\end{aligned}
$$

Due to the parameter estimate $\hat{\theta}_{N}$ is the solution to the normal equation, it means that. 


$$
\begin{aligned}
& \frac{1}{N} \sum_{t=1}^{N} \varphi\left(t, \hat{\theta}_{N}\right) \mathcal{\varepsilon}\left(t, \hat{\theta}_{N}\right)= \\
& \frac{1}{N} \sum_{t=1}^{N} \varphi\left(t, \hat{\theta}_{N}\right)\left[y(t)-\hat{y}\left(t, \hat{\theta}_{N}\right)\right]=0
\end{aligned}
$$

As SPS method builds the confidence region by perturb equation and exploits the information in the data as much as possible, while assuming minimal prior statistical knowledge on the noise [23]. In the whole SPS method, (m-1) sign-perturbed sums are introduced.

$$
\begin{aligned}
& H_{i}(\theta)=\sum_{t=1}^{N} \varphi(t, \theta) \alpha_{i t} \varepsilon(t, \theta)= \\
& \sum_{t=1}^{N} \varphi(t, \theta) \alpha_{i t}[y(t)-\hat{y}(t, \theta)], i=1,2 \cdots m-1
\end{aligned}
$$

where $\left\{\alpha_{i t}\right\}$ are random signs, i.e. independent and identically distributed random variables which takes on the values \pm 1 with equal probabilities $\frac{1}{2}$. When no sign perturbations are used, the reference sum is defined.

$$
H_{0}(\theta)=\sum_{t=1}^{N} \varphi(t, \theta) \varepsilon(t, \theta)
$$

Then SPS method use to construct non-asymptotic confidence region is dependent on the following sums.

$$
\left\{\begin{array}{l}
S_{0}(\theta)=\Phi^{-\frac{1}{2}}(\theta) \sum_{t=1}^{N} \varphi(t, \theta) \varepsilon(t, \theta) \\
S_{i}(\theta)=\Phi^{-\frac{1}{2}}(\theta) \sum_{t=1}^{N} \varphi(t, \theta) \alpha_{i t} \varepsilon(t, \theta)
\end{array}\right.
$$

where $i=\{1,2 \cdots m-1\}$ and matrices $\Phi(\theta)$ are perturbed covariance estimates.

$$
\left\{\begin{array}{l}
\Phi(\theta)=\sum_{t=1}^{N} \varphi(t, \theta) \varphi^{T}(t, \theta) \\
\Phi^{\frac{1}{2}}(\theta) \Phi^{\frac{1}{2}}(\theta)^{T}=\Phi(\theta)
\end{array}\right.
$$

The above sums (35) coming from [21] are used to construct non-asymptotic confidence region for only linear regression model, not our closed loop system. To ensure SPS method suit for closed loop system, some modifications must be added to achieve this goal. The one step ahead prediction is modified, after considering random signs.

$$
\hat{y}\left(t, \theta, \alpha_{i}\right)=G(z, \theta) u\left(t, \theta, \alpha_{i}\right)+H(z, \theta)\left(\alpha_{i t} e(z, \theta)\right)
$$

Then using the feedback structure, input signal on plant is changed as.

$$
u\left(t, \theta, \alpha_{i}\right)=r(t)-K(z) \hat{y}\left(t, \theta, \alpha_{i}\right)
$$

Computing the one step ahead prediction error or residual, with the random signs.

$$
\varepsilon\left(t, \theta, \alpha_{i}\right)=y(t)-\hat{y}\left(t, \theta, \alpha_{i}\right)
$$

Comparing equations (37), (38), (39) and their corresponding expressions, the difference is that here random signs $\alpha_{i t}$ are immerged into their forms respectively, i.e. their relations are listed as.

$$
\left\{\begin{array}{l}
\varepsilon(t, \theta) \rightarrow \varepsilon\left(t, \theta, \alpha_{i}\right) \\
\hat{y}(t, \theta) \rightarrow \hat{y}\left(t, \theta, \alpha_{i}\right) \\
u(t, \theta) \rightarrow u\left(t, \theta, \alpha_{i}\right)
\end{array}\right.
$$

where $\alpha_{i}$ denotes the vector $\left(\alpha_{i 1}, \alpha_{i 2} \cdots \alpha_{i m}\right)$, and $\alpha_{i t}$ is defined as above.

Based on these three modifications about $\varepsilon\left(t, \theta, \alpha_{i}\right)$, $\hat{y}\left(t, \theta, \alpha_{i}\right), u\left(t, \theta, \alpha_{i}\right)$, the reference sum and (m-1) sign-perturbed sums can be evaluated for closed loop system.

$$
\begin{aligned}
& \left\{\begin{array}{l}
S_{0 c}(\theta)=\Phi^{-\frac{1}{2}}\left(\theta, \alpha_{i}\right) \sum_{t=1}^{N} \varphi\left(t, \theta, \alpha_{i}\right) \varepsilon\left(t, \theta, \alpha_{i}\right) \\
S_{i c}(\theta)=\Phi^{-\frac{1}{2}}\left(\theta, \alpha_{i}\right) \sum_{t=1}^{N} \varphi\left(t, \theta, \alpha_{i}\right) \alpha_{i t} \varepsilon\left(t, \theta, \alpha_{i}\right)
\end{array}\right. \\
& \varphi\left(t, \theta, \alpha_{i}\right)=-\frac{\partial \varepsilon\left(t, \theta, \alpha_{i}\right)}{\partial \theta}=\frac{\partial \hat{y}\left(t, \theta, \alpha_{i}\right)}{\partial \theta}
\end{aligned}
$$

Also the perturbed covariance estimate is that.

$$
\Phi\left(\theta, \alpha_{i}\right)=\sum_{t=1}^{N} \varphi\left(t, \theta, \alpha_{i}\right) \varphi^{T}\left(t, \theta, \alpha_{i}\right)
$$

Combing modifications (37)-(42), the non-asymptotic confidence region is constructed by modified SPS method, where the main procedures are formulated as.

1. Given two integer parameters $m>q>0$, such that

$$
p=1-\frac{q}{m}
$$

and $p \in(0,1)$ is a confidence probability.

2. Generate $\mathrm{N}(\mathrm{m}-1)$ independent and identically distributed random signs $\left\{\alpha_{i t}\right\}$ with

$$
P\left(\alpha_{i t}=1\right)=P\left(\alpha_{i t}=-1\right)=\frac{1}{2}
$$

for $i=\{1,2 \cdots m-1\}$, and $i=\{1,2 \cdots N\}$ 
3. For the given $\theta$, compute the prediction outputs, inputs and errors.

$$
\left\{\begin{aligned}
\hat{y}\left(t, \theta, \alpha_{i}\right) & =G(z, \theta) u\left(t, \theta, \alpha_{i}\right) \\
& +H(z, \theta)\left(\alpha_{i t} e(z, \theta)\right) \\
u\left(t, \theta, \alpha_{i}\right) & =r(t)-K(z) \hat{y}\left(t, \theta, \alpha_{i}\right) \\
\varepsilon\left(t, \theta, \alpha_{i}\right) & =y(t)-\hat{y}\left(t, \theta, \alpha_{i}\right)
\end{aligned}\right.
$$

4. Calculate the gradient of $\varepsilon\left(t, \theta, \alpha_{i}\right)$ and the perturbed covariance estimate.

$$
\left\{\begin{array}{l}
\varphi\left(t, \theta, \alpha_{i}\right)=-\frac{\partial \varepsilon\left(t, \theta, \alpha_{i}\right)}{\partial \theta}=\frac{\partial \hat{y}\left(t, \theta, \alpha_{i}\right)}{\partial \theta} \\
\Phi\left(\theta, \alpha_{i}\right)=\sum_{t=1}^{N} \varphi\left(t, \theta, \alpha_{i}\right) \varphi^{T}\left(t, \theta, \alpha_{i}\right)
\end{array}\right.
$$

5. Calculate the factor $\Phi^{-\frac{1}{2}}\left(\theta, \alpha_{i}\right)$ such that.

$$
\Phi^{-\frac{1}{2}}\left(\theta, \alpha_{i}\right) \Phi^{-\frac{1}{2}}\left(\theta, \alpha_{i}\right)^{T}=\Phi\left(\theta, \alpha_{i}\right)
$$

6. Compute the reference sum and (m-1) sign-perturbed sums and compute the rank $R(\theta)$ of $\left\|S_{0 c}(\theta)\right\|^{2}$ as the smallest in the ordering of the variables $\left\|S_{i c}(\theta)\right\|^{2}$

7. Construct non-asymptotic confidence region as

$$
\hat{\Theta}_{N}=\{\theta: R(\theta) \leq m-q\}
$$

One important advantage of SPS method is that the confidence region constructed by equation (48) in closed loop system, has exact confidence probability for any finite data points. The confidence probability of the constructed confidence region is exact $p$, that is

$$
P\left\{\theta \in \hat{\Theta}_{N}\right\}=1-\frac{q}{m}=p
$$

It means that this non-asymptotic confidence region has exact confidence probability as the number of data points is finite

\section{Simulation Example}

To prove the model structure validation strategies under closed loop condition, we consider one simulation system.

$$
\begin{aligned}
y(t) & =G_{0}(z) R(z) w(t)-G_{0}(z) K(z) y(t) \\
& +H_{0}(z) e(t)
\end{aligned}
$$

where $G_{0}(z), H_{0}(z)$ and $K(z)$ are assumed to be as follows respectively.

$$
\begin{aligned}
& G_{0}(z)= \\
& \frac{0.01293 z^{-1}+0.1062 z^{-2}+0.1058 z^{-3}+0.01279 z^{-4}}{1-0.2482 z^{-1}+1.091 z^{-2}-0.2441 z^{-3}+0.9822 z^{-4}} \\
& H_{0}(z)=1, \quad K(z)=1
\end{aligned}
$$

Here the noise model is 1 and it shows the external disturbance acting on closed loop reduces to the white noise disturbance. The feedback is the common positive feedback. The noise $e(t)$ is a white noise with zero mean and unit variance, input $w(t)$ is similar to noise $e(t)$. In order to analyze the confidence region of the model parameter and cross correlation function, we set shaping filter as $R(z)=1$.

The parameterized plant model $G(z, \theta)$ is described as that.

$$
G(z, \theta)=\frac{\theta_{1} z^{-1}+\theta_{2} z^{-2}+\theta_{3} z^{-3}+\theta_{4} z^{-4}}{\theta_{5}+\theta_{6} z^{-1}+\theta_{7} z^{-2}-\theta_{8} z^{-3}+\theta_{9} z^{-4}}
$$

The nine unknown parameters is used to construct the unknown parameter vector $\theta$, i.e. it means that.

$$
\theta=\left[\begin{array}{lllllllll}
\theta_{1} & \theta_{2} & \theta_{3} & \theta_{4} & \theta_{5} & \theta_{6} & \theta_{7} & \theta_{8} & \theta_{9}
\end{array}\right]^{T}
$$

Choose the number of observed data set $\{y(t), w(t)\}_{t=1}^{N}$ as $N=500$, and apply direct approach to identify the unknown parameters in parameterized plant model $G_{0}(z)$. The qualities of nine model parameters affect the output response directly. So the identification accuracy or credibility of model parameters can be all measured by the output response of closed loop.

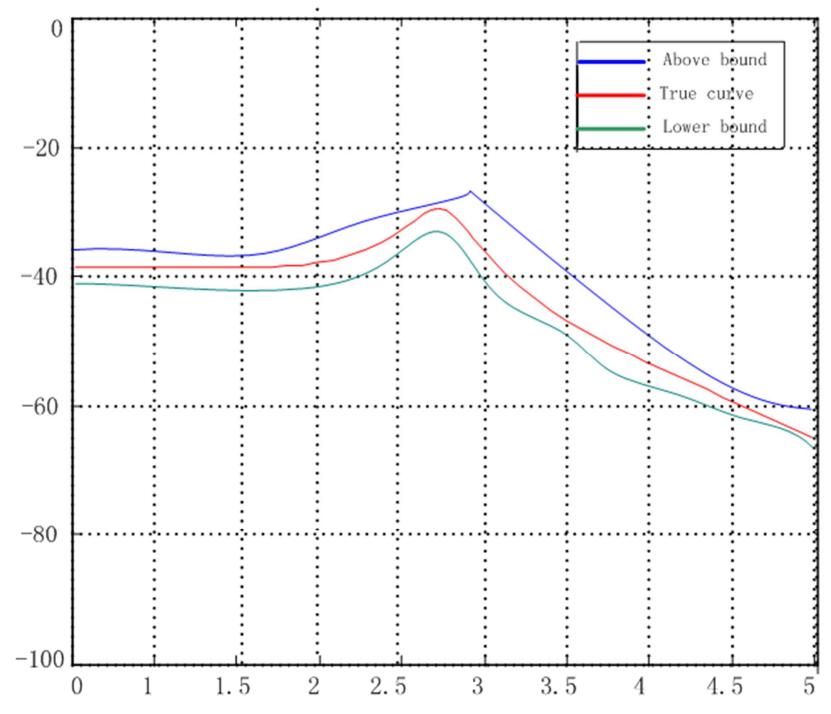

Figure 2. Confidence region of amplitude in Bode plot. 


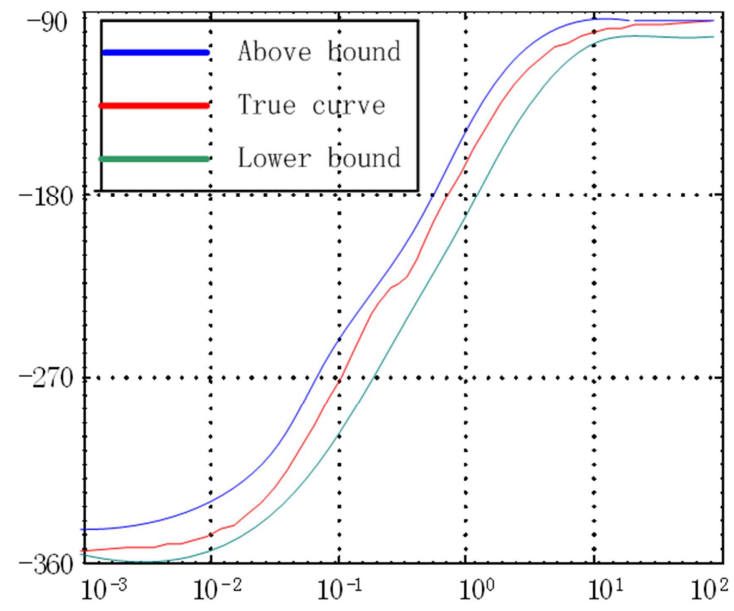

Figure 3. Confidence region of phase in Bode plot.

The whole output frequency response curves are showed in Figure 2, based on estimated model parameters. The red curve is the actual true amplitude curve from Bode plot tool. When the estimated model parameters are contained in the uncertainty bound with probability level 0.99 , the amplitude curves lie above or low the red curve. From Figure 1, we see these three curves are very close and the red amplitude curve lies between two confidence amplitude curves with probability level 0.99 .

Using Matlab simulation tool to simulate the output response of Bode plot in closed loop, the phase plot is get with amplitude plot simultaneously. The confidence region phase plot is given in Figure 3, and the red phase curve lies also between two confidence phase curves with the probability level 0.99. This is similar to the derivation of Figure 2.

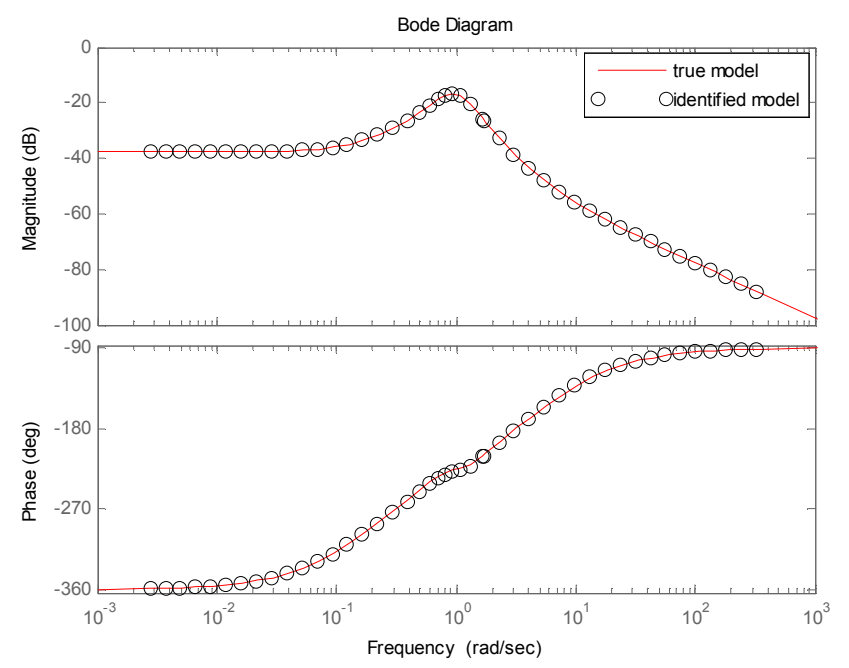

Figure 4. Comparing of the true model and its identified model.

To verify the efficiency of the identified model $G\left(\hat{\theta}_{N}\right)$ and make sure that this identified model can be used to replace the true model, we compare the Bode responses through true model $G_{0}(z)$ and its identified model $G\left(\hat{\theta}_{N}\right)$ respectively in Figure 4. From Figure 4, we see that these two Bode response curves coincide with each other, it means that the model error $\tilde{G}(z)$ will converge to zero with time increases.

\section{Conclusion}

In this paper, the model structure validation problem is analyzed for closed loop condition from two aspects:

(1) asymptotic confidence region,

(2) non-asymptotic confidence region.

Asymptotic system theory is obtained to construct asymptotic confidence region as the number of data points is infinite, and SPS method is modified to construct guaranteed non-asymptotic confidence region for any finite data set. The next subject is to construct one virtual variable to reformulate this asymptotic variance matrix as one projection form of this virtual variable along some vector space?

\section{References}

[1] Robust optimal experiment design for system identification

[2] An optimal structure selection and parameter design approach for a dual-motor-driven system used in an electric bus

[3] The spectral parameter estimation method for parameter identification of linear fractional order systems

[4] Subspace identification and predictive control of batch particulate processes

[5] Urban Forssel, Lennart Ljung, "Closed loop identification revisted," Automatica, vol. 35, no. 7, pp. 1215-1241, 1999.

[6] Ljung, L, "System identification: Theory for the user," Prentice Hall, 1999.

[7] Pintelon R, Schoukens J, "System identification: a frequency domain approach,” New York: IEEE Press, 2001.

[8] Juan C, Augero, "A virtual closed loop method for closed loop identification," Automatica, vol 47, no. 8, pp. 1626-1637, 2011.

[9] U, Forssell, L Ljung, "Some results on optimal experiment design," Automatica, vol 36, no. 5, pp. 749-756, 2000.

[10] M, Leskers, "Closed loop identification of multivariable process with part of the inputs controlled," International Journal of Control, vol 80, no. 10, pp. 1552-1561, 2007.

[11] Hakan Hjalmarssion, "From experiment design to closed loop control," Automatica, vol 41, no. 3, pp. 393-438, 2005,.

[12] Hakan Hjalmarssion, "Closed loop experiment design for linear time invariant dynamical systems via LMI," Automatica, vol 44, no. 3, pp. 623-636, 2008.

[13] X, Bombois, "Least costly identification experiment for control," Automatica, vol 42, no. 10, pp. 1651-1662, 2006.

[14] Roland Hildebrand, "Identification for control: optimal input design with respect to a worst case gap cost function," SIAM Journal of Control Optimization, vol 41, no. 5, pp. 1586-1608, 2003. 
[15] M, Gevers, "Identification of multi input systems: variance analysis and input design issues," Automatica, vol 42, no.410, pp. 559-572, 2006.

[16] M, Gevers, "Identification and information matrix: how to get just sufficiently rich," IEEE Transactions on Automatic control, vol 54, no.12, pp. 2828-2840, 2009.

[17] Graham C Goodin, "Bias issues in closed loop identification with application to adaptive control," Communications in Information and Systems, vol 2, no.4, pp. 349-370, 2002.

[18] James S Welsh, "Finite sample properties of indirect nonparametric closed loop identification," IEEE Transactions on Automatic control, vol 47, no.8, pp. 1277-1291, 2002.

[19] Sippe G Douma, "Validity of the standard cross correlation test for model structure validation," Automatica, vol 44, no.4, pp. 1285-1294, 2008.
[20] Wang Jian-hong, Wang Yan-xiang. "Model structure validation for closed loop system identification," International Journal of Modelling, Identification and Control, vol 27, no.4, 323-331, 2017.

[21] Balazs Cs Cgaji, "Sign perturbed sums: a new system identification approach for constructing exact non-asymptotic confidence region in linear regression models,"IEEE Transactions on Signal Processing, vol 69, no.1, pp. 169-181, 2015.

[22] Scador Kolumhan, Istran Vajk, "Perturb data sets methods for hypothesis testing and structure of corresponding confidence sets," Automatica, vol 51, no.1, pp. 326-331, 2015.

[23] Michel Kieffer, Eric Walter, "Guaranteed characterization of exact non-asymptotic confidence regions as defined by LSCR and SPS," Automatica, vol 50, no.2, pp. 507-512, 2014. 